\section{AB0502 DRUG SURVIVAL ANALYSIS OF TOFACITINIB IN PATIENTS WITH RHEUMATOID ARTHRITIS}

Y. Karakoc ${ }^{1}$, I. Ercan ${ }^{2} .{ }^{1}$ Rheumatology Clinic, Biyofiz Medical Centre;

${ }^{2}$ Bioistatistics, Uludag University Medical Faculty, Bursa, Turkey

Background: The drug survival rate of tofacitinib in patients with Rheumatoid Arthritis (RA) has not been reported so far.

Objectives: To determine the tofacitinib drug survival rate and the factors that may affect it in patients with RA from a single rheumatology clinic.

Methods: We have retrospectively analysed the track records of RA patients to whom tofacitinib was prescribed between June 2014 to December 2017. Descriptive analysis includes sex, duration of disease, autoantibody association, smoking, major trauma exposure, initiation in DMARD/Anti-TNF resistant patients, monotherapy/use in combination with DMARD. According to the duration of the disease, the patients were grouped as early ( $0-4$ year), established (5-10 year), and late RA ( $>10$ year).

Drug survival was estimated using Kaplan-Meier survival analysis, and the independent variables that may affect the discontinuation were investigated by log rank test and modelled by Backward Stepwise Cox regression analysis.

Tofacitinib was prescribed to patients who were resistant to at least three different types of csDMARDs. Low- dose Steroid (below $10 \mathrm{mg}$ ) and NSAID drugs were used as needed.

Results: During the study period, $192(163 \mathrm{~F}, 85 \%)$ patients were prescribed tofacitinib in our clinic. Median age was $56^{21-81}$ years, the median age at onset was $45^{6-72}$ years, and median disease duration was $10^{1-40}$ years. In this study, the ratio of RF and anti-CCP positivity were $63 \%$ and $60 \%$, respectively. $33 \%$ of patients were seronegative. The patients with a smoking history were $26 \%$, and exposure to major trauma was $16 \%$. $15 \%$ of patients were early, $31 \%$ established, and $54 \%$ late $\mathrm{Ra}$.

Tofacitinib was prescribed in $92(48 \%)$ bio-naive and 100 (52\%) bio-experienced patients. It was used as monotherapy in $112(58 \%)$ and in combination with csDMARDs 80 (42\%).

The drug survival rates in Kaplan Meier analysis were $77 \%$ at $3 \mathrm{rd}, 69 \%$ at 6 th, $62 \%$ at 12 th, $54 \%$ at 18 th and $49 \%$ at 24 th, $49 \%$ at 30 th months. Tofacitinib was discontinued in 51 (27\%) patients due to no response and in $22(11 \%)$ patients due to side effects. None of the independent variables in regression analysis showed a relationship to tofacitinib discontinuation $(p>0.05)$. During the follow-up period, one patient had breast cancer, and one had recurrent pneumonia. There were no tuberculosis or shingles cases reported. Two patients died from pulmonary thromboembolism.

Conclusions: We found that drug survival rates of tofacitinib in RA patients were $77 \%$ at 3rd month, $69 \%$ at 6 th month, $62 \%$ at 12 thmonth, $54 \%$ at 18 th month and $49 \%$ at 24 th month, $49 \%$ at 30th months. The main cause of discontinuation of the drug was inefficiency and the loss of efficiency. We could not find any link between the predetermined independent variables and the drug discontinuation. This result raises questions about why the drug loses its efficacy in some patients in time, and how this could be preventable.

Acknowledgements: One of the theories of autoimmunity is that Damage -Associated Molecular Patterns (DAMPs) may give rise to autoimmune inflammation. We were curious about how many of patients suffered from major trauma, which was defined as accidents terminated with fractures and dislocations or falls from a height of at least three metres.

Disclosure of Interest: None declared

DOI: 10.1136/annrheumdis-2018-eular.1893

\section{SLE, Sjögren's and APS - treatment}

\section{AB0503 \\ THERAPEUTIC STRATEGY AND SHORT-TERM OUTCOME IN NEUROPSYCHIATRIC SYSTEMIC LUPUS ERYTHEMATOSUS}

A. Bortoluzzi ${ }^{1}$, A. Fanouriakis ${ }^{2}$, S. Appenzeller ${ }^{3}$, L. Carli ${ }^{4}$, G. Carrara ${ }^{5}$, F. Conti ${ }^{6}$,

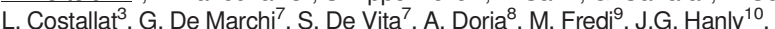

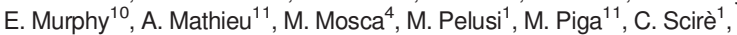

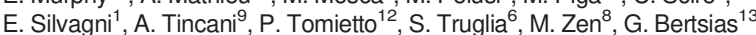
M. Govoni' . ${ }^{1}$ University of Ferrara, Cona (Ferrara), Italy; ${ }^{2}$ Attikon University Hospital, Athens, Greece; ${ }^{3}$ State University of Campinas, Campinas, Brazil; ${ }^{4}$ University of Pisa, Pisa; ${ }^{5}$ Epidemiology Unit, Italian Society of Rheumatology, Milan; ${ }^{6}$ Sapienza University of Rome, Rome; ${ }^{7}$ University of Udine, Udine; ${ }^{8}$ University of Padova, Padova; ${ }^{9}$ University of Brescia, Brescia, Italy, ${ }^{10}$ Dalhousie University, Halifax, Nova Scotia, Canada; ${ }^{11}$ University of Cagliari, Cagliari; ${ }^{12} \mathrm{AOU}$ Ospedali Riuniti of Trieste, Trieste, Italy, ${ }^{13}$ University of Crete, Heraklion, Greece

Background: The treatment of neuropsychiatric systemic lupus erythematosus (NPSLE) is extremely challenging and only a few clinical trials have been performed to establish optimal management.
Objectives: To describe the therapeutic approach and the short-term outcome of a multi-centre cohort of patients with NPSLE, enrolled at the time of the first NP event.

Methods: This is a retrospective cohort study. All NP events were defined according to American College of Rheumatology (ACR) case definition and divided into 3 clusters: central/diffuse $(C / D)$, central/focal $(C / F)$ and peripheral $(P)$. A validated attribution algorithm was used to determine the attribution of all NP events. Demographic variables, global SLE disease activity Index 2000 (SLEDAI$2 \mathrm{~K}$ ), cumulative organ damage (SLICC/ACR Damage Index (SDI)) and treatment adopted for NP manifestations were collected. The clinical outcome of all NP events was determined by a physician-completed seven-point Likert scale (1=patient demise, 2 =much worse, 3 =worse, $\quad 4=$ no change, 5 =improved $6=$ much improved, $7=$ resolved). The relationship between the variables of interest and the outcome was analysed by crude and adjusted logistic models and reported as Odds Ratio (OR) and $95 \%$ confidence intervals $(95 \% \mathrm{Cl})$.

Results: 461 SLE patients with at least one NP event were included. $91.8 \%$ o patients were female, mean (SD) age 35.4 (13.6) years. $19.7 \%(91)$ of events were observed at diagnosis of SLE, $13.4 \%(62)$ before and $66.8 \%(308)$ after the diagnosis. 111 events (24.1\%) were C/F, 286 (62\%) C/D and 64 (13.9\%) P. 198 $(42.95 \%)$ of all NP events were attributed to SLE. The overall probability of immunosuppressive therapy was $28.4 \%(95 \% \mathrm{Cl} 24.3-32.8), 38.7 \%(95 \% \mathrm{Cl} 29.6-$ 48.5) in $\mathrm{C} / \mathrm{F}, 21.3 \%(95 \% \mathrm{Cl} 16.7-26.5)$ in $\mathrm{C} / \mathrm{D}$ and $42.2 \%(95 \% \mathrm{Cl} 16.7-26.5)$ in $\mathrm{P}$ manifestations. The probability of immunosuppressive therapy was $47.9 \%$ (95\% Cl 40.8-55.2) in attributed events. The one-year outcome was available in 355 patients. Physician assessment indicated resolution (76 patients) or improvement (150 patients) in 49\% (226/461) of cases. The crude and adjusted OR of attributed NP events and immunosuppressants on a favourable outcome is illustrated in Figure 1. The multivariable logistic regression analysis was done adjusting for age at diagnosis of SLE [OR 0.96, 0.94-0.98] $p=0.001$, female gender [OR $0.97,0.33$ 2.7] $p=0.959$, SDI $[0.85,0.68-1.08] p=0.202$, SLEDAI-2K $[1.06,1.01-1.11]$ $\mathrm{p}=0.008$ and type of event (F/C [REF], $C / D[0.37,0.16-0.83] \mathrm{p}=0.016, \mathrm{P}[0.54$, $0.21-1.42] p=0.215)$.

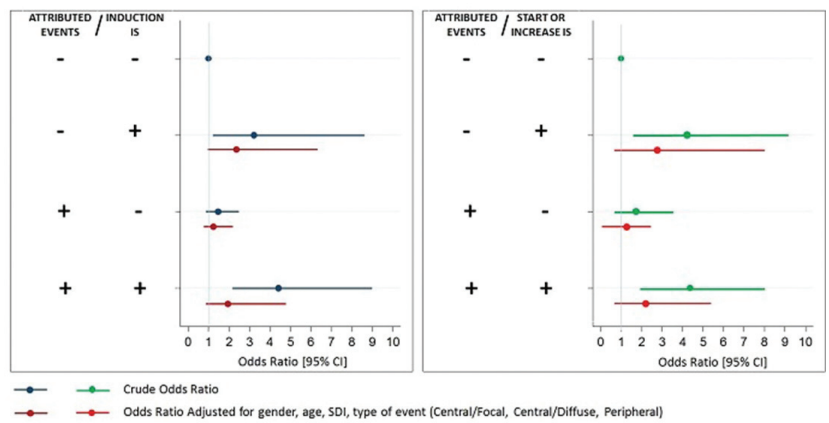

Abstract AB0503 - Figure 1. Crude and adjusted OR of attributed NP events and induction immunosuppression (IS, Figure 1A) or start/increase IS (Figure 1B) on a favourable outcome

Conclusions: In our study, the therapeutic immunosuppressive approach was mostly used in attributed, C/F and $\mathrm{P}$ manifestations. In patients treated with immunosuppressants, the favourable outcome was lower in C/D phenotype.

Disclosure of Interest: None declared

DOI: 10.1136/annrheumdis-2018-eular.6854

\section{AB0504 EFFICACY OF PROLONGED MAINTENANCE MONOTHERAPY WITH RITUXIMAB IN PATIENTS WITH PRIMARY SJÖGREN'S SYNDROME: THREE-YEAR FOLLOW-UP}

A. Torgashina ${ }^{1}$, E. Rodionova ${ }^{1}$, T. Safonova ${ }^{2}$, V. Vasiliev ${ }^{1} .{ }^{1}$ Nasonova Research $^{2}$ Institute of Rheumathology; ${ }^{2}$ Research Institute of Ophthalmic Diseases, Moscow, Russian Federation

Background: There are currently no effective systemic therapies of primary Sjögren's syndrome (pSS); however, open label series have suggested that rituximab may be beneficial for systemic and glandular manifestations.

Objectives: To estimate clinical efficacy and safety of prolonged maintenance B cell targeted monotherapy for pSS.

Methods: 25 with pSS ACR-EULAR criteria, 2016 were included in this research. Indications for treatment were significant immunological activity (high titres of rheumatoid factor (RF) and anti-nuclear antibodies) and/or hypergammaglobulinemia in 20 patients, parotid enlargement (lymphoma was excluded) - 5, arthritis -3 , lymphadenopathy -3 , severe keratoconjunctivitis sicca in 7 , of them corneal 